\title{
Theoretical Assessment of Hinge-Type Models for Electron Donors in Reaction Centers of Photosystems I and II as Well as of Purple Bacteria
}

\author{
Denis G. Artiukhin ${ }^{\dagger 1}$, Patrick Eschenbach॥ , Jörg Matysik ${ }^{\ddagger}$, \\ and Johannes Neugebauer ${ }^{\| 2}$ \\ † Department of Chemistry, Aarhus Universitet, DK-8000 Aarhus, Denmark \\ \| Theoretische Organische Chemie, Organisch-Chemisches Institut and Center for \\ Multiscale Theory and Simulation, Westfälische Wilhelms-Universität Münster, \\ Corrensstraße 40, 48149 Münster, Germany \\ ¥ Institut für Analytische Chemie, Universität Leipzig, Linnéstr. 3, 04103 Leipzig, \\ Germany
}

Date: November 26, 2020

\footnotetext{
${ }^{1}$ Email: artiukhin@chem.au.dk

${ }^{2}$ Email: j.neugebauer@uni-muenster.de
} 


\begin{abstract}
Hinge-type molecular models for electron donors in reaction centers of Photosystem I, II, and purple bacteria were investigated using a two-state computational approach based on Frozen-Density Embedding. This methodology, dubbed FDE-diab, is known to avoid consequences of the self-interaction error as far as intermolecular phenomena are concerned, which allows to predict qualitatively correct spin densities for large bio-molecular systems. The calculated spin density distributions are in a good agreement with available experimental results and demonstrated a very high sensitivity to changes in relative orientiation of co-factors and amino-acid protonation states. This allows to validate the previously proposed hinge-type models and make predictions on protonation states of axial histidine molecules. Contrary to the reaction centers in Photosystem I and purple bacteria, the axial histidines from Photosystem II were found to be deprotonated. This fact might shed some light on remarkable properties of Photosystem II reaction centers.
\end{abstract}




\section{Introduction}

Reaction centers (RCs) of photosystems I and II (commonly referred to as PSI and PSII) share many structural similarities $[1,2]$. They both are formed by two protein subunits and associated photosynthetic co-factors. The latter have nearly- $C_{2}$ symmetric spacial arrangements and, in the case of PSII, include (but are not limited to) the pairs of i) inner chlorophyll $a$ (Chl $a$ ) co-factors, ii) accessory Chl $a$ pigments, iii) pheophytin $a$ (Phe $a$ ) molecules, and iv) plastoquinones. The first three pairs are labeled as $\mathrm{P}_{\mathrm{D} n}, \mathrm{Chl}_{\mathrm{D} n}$, and $\mathrm{Ph}_{\mathrm{D} n}$, where $\mathrm{D} n$ is the protein subunit they belong to, i.e., D1 or D2. The plastoquinones are often denoted as $\mathrm{Q}_{\mathrm{A}}$ and $\mathrm{Q}_{\mathrm{B}}$. In PSI, the inner pair of co-factors is formed by Chl $a$ and its $\mathrm{C}-13^{2}$-epimer $\left(\mathrm{Chl} a^{\prime}\right)$, which are called $\mathrm{P}_{\mathrm{B}}$ and $\mathrm{P}_{\mathrm{A}}$, respectively. Similar to the case of PSII, the pair of accessory PSI pigments is composed of Chl $a$ molecules (denoted as $\mathrm{A}_{-1 \mathrm{~A}}$ and $\left.\mathrm{A}_{-1 \mathrm{~B}}\right)$, whereas pheophytins and plastoquinones are replaced by Chl $a$ cofactors $\left(\mathrm{A}_{0 \mathrm{~A}}\right.$ and $\left.\mathrm{A}_{0 \mathrm{~B}}\right)$ and phylloquinones $\left(\mathrm{A}_{1 \mathrm{~A}}\right.$ and $\left.\mathrm{A}_{1 \mathrm{~B}}\right)$, respectively. In both PSI and PSII, magnesium ions of inner-pair Chls are coordinated by histidine (His) amino-acid residues, whereas those of accessory pigments are coordinated by water molecules.

Despite these structural similarities, the redox potentials of the primary electron donors P700 in PSI and P680 in PSII (the numbers within their names refer to the absorption maxima) differ by about $0.7 \mathrm{~V}$ and are equal to $+0.5 \mathrm{~V}$ and $+1.2 \mathrm{~V}$, respectively [3]. This makes the donors P700 and P680 the strongest reducing and oxidation agents, respectively, known in living matter. These electron donors are composed of different co-factors [4]: P700 consist of the inner pair Chls, while P680 is a monomeric accessory Chl $a$. Additionally, it is known that after photo-excitation PSII performs one-sided electron transfer (ET) along the protein subunit D1 [5-7], while a two-sided ET is observed in PSI $[8,9]$. These facts pose the question, which factors cause the difference in functional symmetry break. 
The method of solid-state Photochemically Induced Dynamic Nuclear Polarization (PhotoCIDNP) Nuclear Magnetic Resonance (NMR) is especially attractive for studying the electron donors in photosynthetic systems as it provides an access to their electronic structures and can be applied to RCs in living cells of bacteria [10] and plants as well as to whole plants [11]. Using this approach, chemical shifts of photosynthetic pigments associated with the electronic ground-state can be measured [12,13]. The electronic spin-density distribution within the radical-pair state is related, although in a complex fashion [14], to photo-CIDNP intensities. Moreover, considering electronic structures of (bacterio)chlorophyll [(B)Chl] co-factors in terms of simplified frontier orbital models, the spatial distribution of highest occupied molecular orbitals can be reconstructed [15], whereas electron-spin densities measured for the donor triplet state can be used to access spin-density contributions from the lowest unoccupied molecular orbital [16] within similar model assumptions.

${ }^{13} \mathrm{C}[17,18]$ and ${ }^{15} \mathrm{~N}[3]$ photo-CIDNP MAS NMR measurements carried out for the electron donor in PSII revealed a strongly asymmetric (compared to Chl $a$ in solution [19]) electron spin-density distribution and led to the formulation of the so-called hinge model in Ref. [3]. According to this model, the strong spin-density shift is caused by the coordinated histidine (His) molecule, which is deprotonated at the $\pi$-N atom (for nomenclature, see Fig. 1) and, therefore, is negatively charged. Thus, the electron donor in its dark state appears to be the anion $[\mathrm{Chl} \cdots \text { His }]^{-}$, which is oxidized to $[\mathrm{Chl} \cdots \mathrm{His}]^{\bullet}$ in the charge-separation event. The His moiety can slightly bend towards pyrrole ring $D$ of the Chl $a$ co-factor (Fig. 1) increasing the $\pi-\pi$ overlap of the conjugated systems and, as a result, inducing shifts of the spin-density into ring $D$ and into the aromatic ring of the His residue [3]. Additionally, it was proposed that the distance between Chl $a$ and His can also be changing depending on the redox state of the complex. Earlier ENDOR measurements [20] indicated that Chl a molecules of the inner pair are weakly coupled and about $82 \%$ of the spin density is localized at a single Chl $a$ molecule. Thus, according 
to the hinge model of the electron donor, a part of the remaining contribution is localized at the His molecule.

The quantum-chemical assessment of such models is an extremely challenging task as it usually requires considering the nearby protein environment. Hence, the choice of a quantum chemical method is practically limited to the standard Kohn-Sham Density Functional Theory (KS-DFT) or its variations such as, for example, Frozen-Density Embedding (FDE) [21]. However, KS-DFT is known to suffer from the self-interaction error (SIE) [22-24], which leads to overdelocalized spin densities [25, 26]. Although reliable spin distributions can often be obtained for single molecules (for example, see Ref. [27]), molecular systems containing non-covalently bonded fragments, similar to those in RCs, could be especially problematic and lead to inconclusive results [26,28]. A methodology allowing to effectively avoid this limitation was reported in our recent work and called FDE-diab [28]. It was tested on model molecular clusters featuring different degrees of spin-density delocalizations and compared to accurate ab initio computations [28]. More recently, it was also applied to inner pair co-factors in RCs of PSI, PSII, and purple bacteria, and verified against previously available experimental measurements [29]. In both cases, a good agreement with theoretical and experimental results was found proving that the suggested approach is robust and effective. It should also be noted that this approach allows to consider much larger molecular systems then accessible with KS-DFT. This makes it a very attractive tool for investigations of photosynthetic pigment models in RCs.

In this work, we apply the FDE-diab methodology to reliably calculate spin-density distributions and to assess the hinge model for the donor in PSII. To that end, we calculate a complex of the radical cation $[\mathrm{Chl} a]^{+\bullet}$ with both protonated and deprotonated axial His moiety for different intermolecular angles and displacements. For comparison purposes, we repeat similar computations for hinge-like models from PSI and the bacterial RC of 
the purple bacterium Rhodobacter sphaeroides. Additionally, we investigate the roles of the protonation state ot the His residue and truncation of the long $\mathrm{Chl} a$ hydrocarbon tail as well as the influence of the surrounding protein environment on the resulting spin distributions.

In what follows, we briefly describe the FDE-diab theory in Sec. 2. Computational details are given in Sec. 3, while results of FDE-diab computations are presented in Sec. 4. Conclusion and outlook are provided in Sec. 5.

\section{Theory}

In this section, we briefly sketch the underlying theory of the FDE-diab approach for two-state models. For a more detailed description, we refer the reader to the original works on FDE-ET [30-33] and to our work on its generalization towards electronic and spin densities [28].

Throughout the paper, we will consider molecular systems composed of two non-covalently bonded molecules $A$ and $B$, which form a charged or neutral radical, e.g., $[A \cdots B]^{+\bullet}$. In these systems, charge and spin are often delocalized between both molecules to some extent. A qualitatively correct description of this delocalization can be achieved by constructing an electronic wave function $\Psi$ as a linear combination,

$$
\Psi=a \Phi_{1}+b \Phi_{2}
$$

where $\Phi_{1}$ and $\Phi_{2}$ are quasi-diabatic electronic wave functions, which correspond to chargeand spin-localized states $\left|A^{+\bullet} \mathrm{B}\right\rangle$ and $\left|A B^{+\bullet}\right\rangle$, while $a$ and $b$ are linear combination coefficients. To construct these quasi-diabatic wave functions, we use the FDE approach [21] and carry out FDE calculations of the radical cations $\left[A^{+\bullet} \cdots B\right]$ and $\left[A \cdots B^{+\bullet}\right]$. The 
Kohn-Sham equations with constrained electronic density (KSCED) [21,34],

$$
\left[-\frac{\nabla^{2}}{2}+v_{\mathrm{KS}}^{I, \sigma}(\vec{r})+v_{\mathrm{emb}}^{I, \sigma}(\vec{r})\right] \psi_{i}^{I, \sigma}(\vec{r})=\epsilon_{i}^{I, \sigma} \psi_{i}^{I, \sigma}(\vec{r}),
$$

are used to compute molecular orbitals (MOs) $\psi_{i}^{I, \sigma}(\vec{r})$ of subsystems $A, B, A^{+\bullet}$, and $B^{+\bullet}$ as well as their energies $\epsilon_{i}^{I, \sigma}$. In Eq. (2), $-\frac{\nabla^{2}}{2}$ is the one-electron kinetic energy operator, $v_{\mathrm{KS}}^{I, \sigma}(\vec{r})$ is the one-electron KS potential, and $v_{\mathrm{emb}}^{I, \sigma}(\vec{r})$ is the one-electron embedding potential. Subsystems are denoted by the superscript $I$, whereas $\sigma=\alpha$ or $\beta$ are spin labels. Calculated MOs are used to construct Kohn-Sham determinants $|A\rangle,|B\rangle,\left|A^{+\bullet}\right\rangle$, and $\left|B^{+\bullet}\right\rangle$. The charge-localized states $\Phi_{1}$ and $\Phi_{2}$ are then obtained as direct products of these determinants $[35,36]$ such that

$$
\Phi_{1} \equiv\left|A^{+\bullet} B\right\rangle=\left|A^{+\bullet}\right\rangle \otimes|B\rangle
$$

and

$$
\Phi_{2} \equiv\left|A B^{+\bullet}\right\rangle=|A\rangle \otimes\left|B^{+\bullet}\right\rangle
$$

Hence, the charge-localized states $\Phi_{1}$ and $\Phi_{2}$ are also Slater determinants consisting of subsystem MOs. Because MOs of different subsystems are not kept orthogonal to each other in FDE, the resulting charge-localized states $\Phi_{1}$ and $\Phi_{2}$ are also non-orthogonal.

In order to find the linear combination coefficients $a$ and $b$ from Eq. (1), a generalized eigenvalue problem in the basis of constructed quasi-diabatic states $\Phi_{1}$ and $\Phi_{2}$ needs to be solved. This requires a construction of $2 \times 2$ overlap $\mathbf{S}$ and Hamilton $\mathbf{H}$ matrices. The elements of $\mathbf{S}$ are equal to determinants of MO overlap matrices with elements $\left\langle\psi_{i}^{(n), \sigma} \mid \psi_{j}^{(m), \sigma}\right\rangle$, whereas the Hamilton matrix elements are calculated as KS energy functionals of scaled transition electron densities. The solution of the generalized eigenvalue problem gives access to two sets of linear combination coefficients (eigenvectors) and two electronic energies $E_{0}$ and $E_{1}$ (eigenvalues) corresponding to the resulting adiabatic electronic states $\Psi_{0}$ and $\Psi_{1}$, respectively. With the adiabatic wave functions available, the 
electronic spin-density $\rho^{\alpha-\beta}(\vec{r})$ of the radical cation can be calculated as

$$
\rho^{\alpha-\beta}(\vec{r})=\frac{\left\langle\Psi\left|\hat{\rho}^{\alpha}-\hat{\rho}^{\beta}\right| \Psi\right\rangle}{\langle\Psi \mid \Psi\rangle} .
$$

The spin-density distributions calculated with FDE-diab methodology were assessed for a number of molecular complexes in Ref. [28] and for inner pairs of (B)Chl molecules in RCs of PSI, PSII, and puple bacteria in Ref. [29] showing good agreements with both accurate theoretical and experimental results.

As described in Refs. [30-33], an additional approximation is often invoked to obtain the electronic coupling between quasi-diabatic states. To this end, the secular determinant is written in a simplified form such that

$$
\operatorname{det}(\mathbf{H}-E \mathbf{S}) \approx\left|\begin{array}{cc}
H_{11}^{\prime}-E & H_{12}-E S_{12}^{\prime} \\
H_{12}-E S_{12}^{\prime} & H_{22}^{\prime}-E
\end{array}\right|=0 .
$$

This enables its analytical solution and gives access to the eigenvalue difference $\Delta E$ (often referred to as excitation energy) [36-38],

$$
\Delta E=\sqrt{\frac{\left(H_{11}^{\prime}-H_{22}^{\prime}\right)^{2}}{1-S_{12}^{\prime 2}}+4 V^{2}},
$$

and the electronic coupling $V$,

$$
V=\frac{1}{1-S_{12}^{\prime 2}}\left[H_{12}-S_{12}^{\prime} \frac{H_{11}^{\prime}+H_{22}^{\prime}}{2}\right] .
$$

As was demonstrated in previous works [30-33], this approximate treatment provides sufficiently accurate values of long-range excitation energies and electronic couplings.

\section{Computational Details}

Calculations presented in this work were carried out for hinge-like molecular models composed of the inner (B)Chl $a$ molecule and the axial His in vacuum and in protein binding 
pockets. We denote these complexes as [Cof $\cdots$ His], where Cof is the $\mathrm{P}_{\mathrm{D} 1}, \mathrm{P}_{\mathrm{A}}$ or $\mathrm{D}_{\mathrm{A}}$ co-factor from RCs of PSII, PSI or purple bacteria, respectively. Subscripts D1 and A are used here to label the protein sub-units to which the co-factors belong. We consider models with i) protonated and deprotonated $\pi-\mathrm{N}$ position of His and ii) the long hydrocarbon tails $\mathrm{R}$ of (B)Chl $a$ truncated and subsequently replaced by $-\mathrm{CH}_{3}$ or left unchanged (for notations, see Fig. 1). In order to distinguish these different complexes, an additional label " $\mathrm{t}$ " is introduced. Thus, the hinge model composed of the truncated $\mathrm{P}_{\mathrm{D} 1}$ and the protonated His is denoted as $\left[\mathrm{tP}_{\mathrm{D} 1} \cdots \mathrm{HisH}\right]$, whereas its deprotonated and untruncated counterpart is written as $\left[\mathrm{P}_{\mathrm{D} 1} \cdots \mathrm{His}\right]^{-}$. In addition to these structural changes, molecular complexes with modified relative orientations of (B)Chl $a$ and His were generated in the vacuum. For the intermolecular distances between the magnesium ion and $\tau$-N of His (see Fig. 1) coordinated to it, values of $\Delta R=0.00, \pm 0.01$ and $\pm 0.03 \AA$ relative to the distances $R$ characteristic to the crystal structure were considered. The angle $\theta$ between the $\tau$-N atom of His, magnesium, and the middle of the (B)Chl $a$ C-17-C-18 bond (see Fig. 1) was modified by $\Delta \theta=0, \pm 5, \pm 10$, and \pm 15 degrees.

Molecular models for hinge-like complexes from PSI, PSII, and bacterial RC were extracted from crystal structures of Synechococcus elongatus [1] (PDB entry 1JB0), Thermococcus vulcanus [2] (PDB entry 3WU2), and Rhodobacter sphaeroides [39] (PDB entry 1M3X), respectively. In case of protein binding pockets, we included all residues within a distance of $4 \AA$ from atoms of a [Cof $\cdots$ His] complex into the model. If at least one atom of a nearby residue appeared within this radius, the entire residue was included into the model. The REDUCE program [40] was used to add missing hydrogen atoms to photosynthetic co-factors, whereas CHARMM22 topology files [41,42] were applied for amino acids. Cut peptide bonds were saturated with neutral capping groups $-\mathrm{C}(\mathrm{O}) \mathrm{CH}_{3}$ and $-\mathrm{NH}_{2}$, where the initial interatomic bond distances were set to average values found in small organic molecules as reported in Ref. [43]. In cases of overlapping capping groups, the missing amino acid residue was added to the model instead. 
Generated dimeric molecular structures in vacuum, i.e., without binding-pocket model, were optimized with the ORCA [44] program package similarly to inner pair models from Ref. [29]: First, positions of hydrogen atoms only were optimized and subsequently intramolecular bond lengths were relaxed, while keeping all the remaining degrees of freedom fixed (for more details, see Ref. [29]). To this end, the BP86 XC functional [45,46] and the def2-TZVP basis set $[47,48]$ were used. The computational cost of structure optimization was reduced by using the resolution-of-the-identity approximation with the auxiliary Coulomb fitting def2-TZV/J basis $[47,48]$. To account for dispersion interactions, the D3BJ correction with Becke-Johnson damping [49,50] were enabled. Binding pockets were initially optimized in a similar fashion as described in Ref. [29]. To this end, we first optimized the positions of hydrogens and then relaxed the atoms of (B)Chl $a$ and His composing the hinge-like complexes within the pocket. This approach, however, led to severe difficulties in SCF convergence during successive FDE calculations as molecular structures of amino acids covalently bond to His were not fully optimized. An alternative approach with (B)Chl $a$, His, and all covalently bond amino acids relaxed led to large changes in the intermolecular orientation compared to the original crystal structure. Therefore, it was decided to replace the long peptide tail connected to the His amino acid with neutral capping groups and, in addition to the two structure optimization steps described above, fully optimize their atoms keeping the remaining molecular structure fixed. The Density Functional Tight Binding (DFTB) method from the AMSDFTB module of the ADF package [51] in conjunction with the parameters set from the Third-Order Parametrization for Organic and Biological Systems (3ob) [52,53], and the dispersion D3BJ correction $[49,50]$ were applied in all three steps. The resulting reduced molecular structures were used without further re-optimization in succeeding spin-density calculations of the corresponding radicals.

Spin-density calculations were carried out using the KS-DFT and FDE-diab [28, 3033] approaches. The ADF program package [51] was applied in both cases. For KS- 

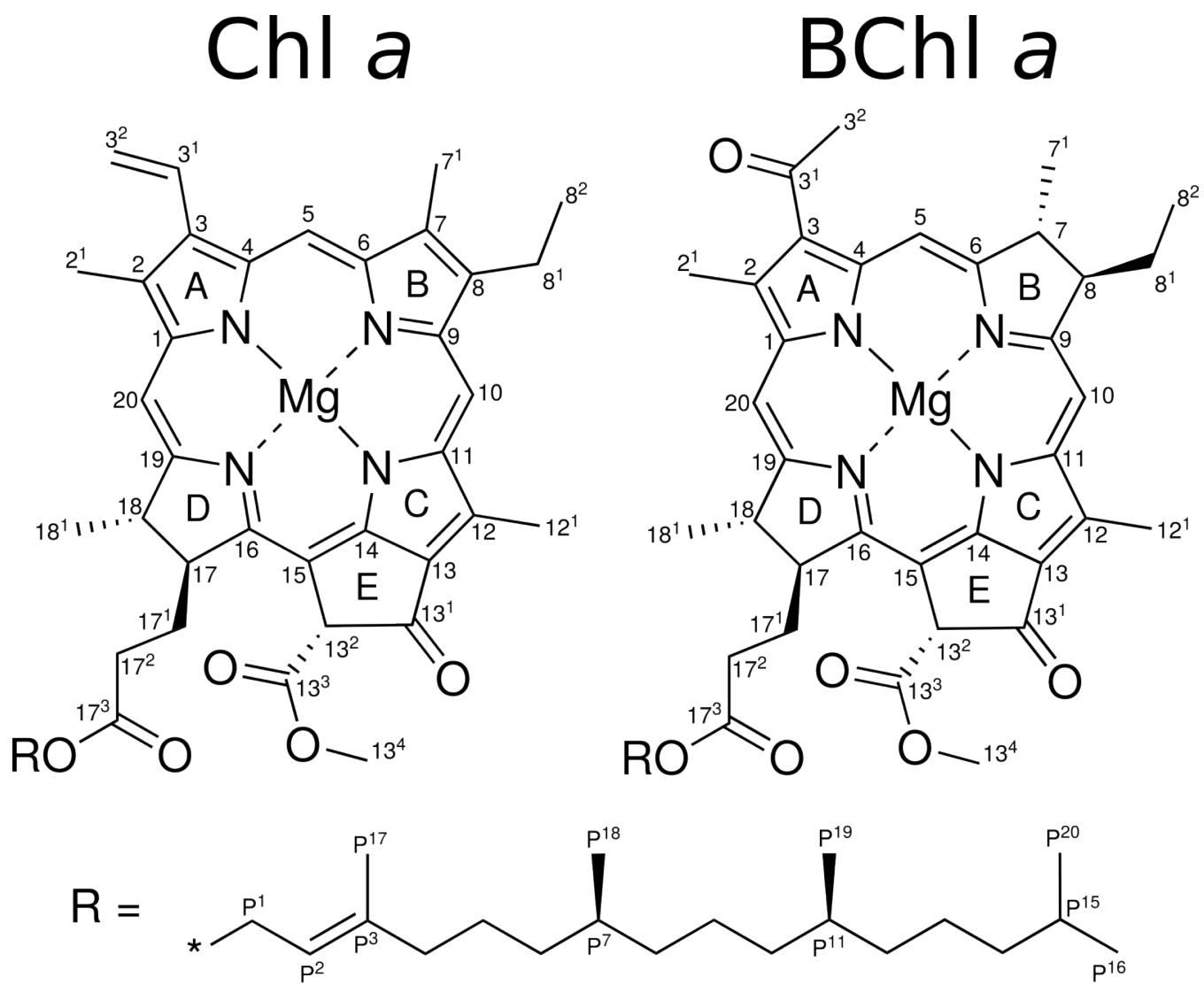

Histidine

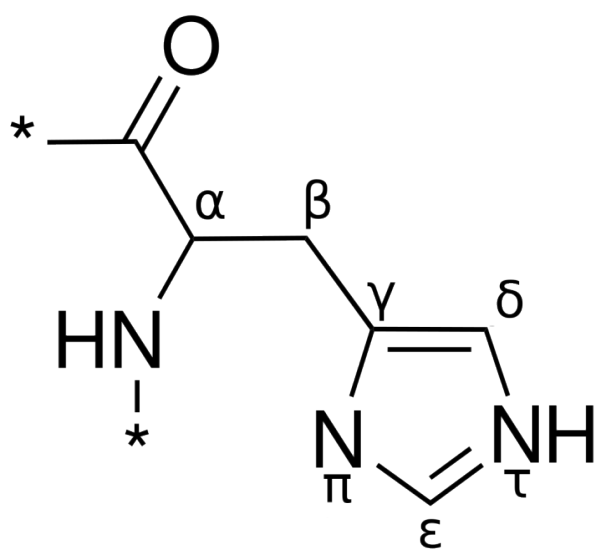

Figure 1: Lewis structures of $\mathrm{Chl} a, \mathrm{BChl} a$, and the histidine molecule protonated at the $\tau$-N position. IUPAC atomic numberings are shown.

DFT, a number of different XC functionals such as Generalized Gradient Approximations (GGA) PW91 [54,55], meta-GGA TPSS [56,57], hybrid B3LYP [58-60] and BHandHLYP, 
meta-hybrid M06-HF [61,62], and B3LYP with the Yukawa range separation (CAMYB3LYP) [63] were employed. The TZP basis set [64] from the ADF program library was consistently applied in all these calculations. In FDE-diab calculations, the initial diabatic states were constructed from MOs obtained from the FDE approach [21] and corresponding to the hinge model molecules. Note that in cases of protein binding pockets, we did not include the MOs of environment molecules into the resulting diabatic states. To this end, modified versions of ADF and PYADF [65] were used. We consistently applied the PW91 [54,55] XC and kinetic-energy PW91k [66] functional in combination with the the TZP basis set [64] in all FDE computations. To relax the subsystem electronic densities (including those of environment molecules), three freeze-and-thaw cycles [67] were employed.

Following the computational protocol developed in our previous works [27-29], we assessed the degree of spin-density delocalization by employing the Becke population analysis (for comparison of Becke, Mulliken, and Bader population analyses, see the Supporting Information [SI] for Ref. [29]). To this end, fine atom-centered Becke grids [68,69] were generated with the SERENITY program [70] and used for spin-density integration over predefined atomic basins. This procedure ensures the error of numerical integration to be below about $5.0 \times 10^{-4}$ atomic units (a.u.). The ratios reported in this work and used as a measure of the spin-density delocalization are computed by summing up the Becke atomic populations over all atoms in a molecule. 


\section{Results}

\subsection{Reaction Center of Photosystem II}

Before proceeding to FDE-diab calculations of spin densities for the hinge model, we demonstrate results of conventional KS-DFT. Calculated spin-density distributions for the $\left[\mathrm{tP}_{\mathrm{D} 1} \cdots \mathrm{His}\right]^{\bullet}$ model are shown in Fig. 2. Although KS-DFT was proven to produce qualitatively correct spin-density distributions for single photosynthetic co-factors with many $\mathrm{XC}$ functionals [27], the results for $\left[\mathrm{tP}_{\mathrm{D} 1} \cdots \mathrm{His}\right]^{\bullet}$ are strongly dependent on the XC approximation used. The non-hybrid functionals PW91 and TPSS as well as the hybrid functionals B3LYP and CAMY-B3LYP (asymptotically 65\% exact exchange) with percentages of exact exchange below $50 \%$ predict most of spin density to be localized on the His molecule. This does not agree with experimental results [20], where about $82 \%$ of the spin density was found at Chl $a$. Increasing the amount of exact exchange to $50 \%$ with BHandHLYP, the spin density can be localized more on the Chl a molecule, whereas completely localized distributions can be found in case of the M06-HF functional (100\% of exact exchange). In turn, however, increasingly large areas of negative spin density and larger spin contaminations are observed $[26,27,30,71-74]$. The latter usually indicates a poor quality of the unrestricted DFT solution. It is interesting to note that calculations for the tilted structures do not show a noticeable change in the internal Chl $a$ spin-density distribution nor in its intermolecular delocalization degree. Therefore, KSDFT calculations are apparently inconclusive and unable to provide useful insight into the spin-density distribution of the hinge model. As was already discussed above, this behavior of the KS-DFT method is not surprising and is related to the SIE [22-24], which often leads to overdelocalized spin densities $[25,26]$.

FDE-diab energy diagrams for the protonated complexes $\left[\mathrm{tP}_{\mathrm{D} 1} \cdots \mathrm{HisH}\right]^{+\bullet}$ and $\left[\mathrm{P}_{\mathrm{D} 1} \cdots \mathrm{HisH}\right]^{+\bullet}$ are presented in Fig. 3, while a detailed list of all calculated values can be 
PW91

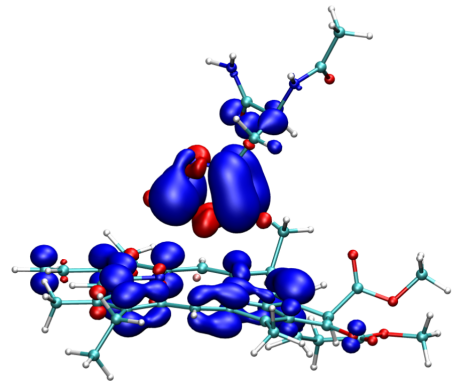

B3LYP

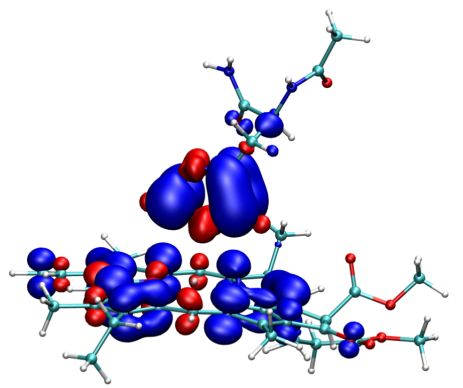

BHandHLYP

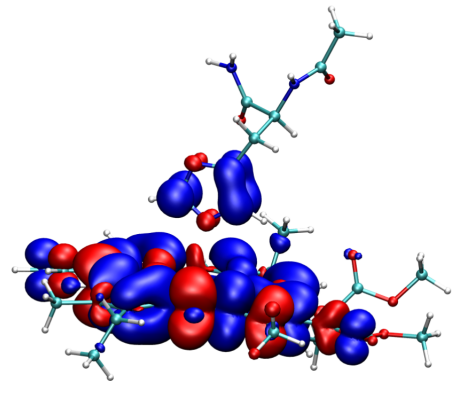

TPSS

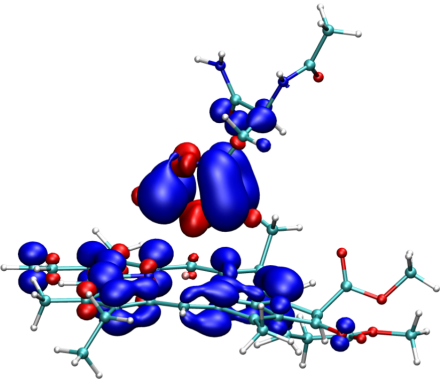

CAMY-B3LYP

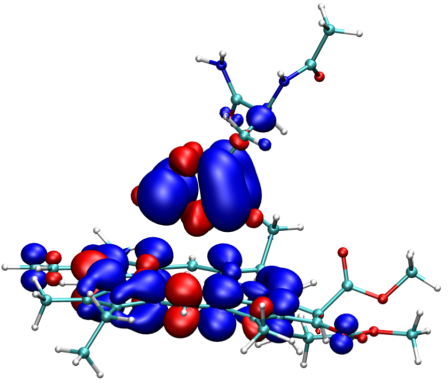

M06-HF

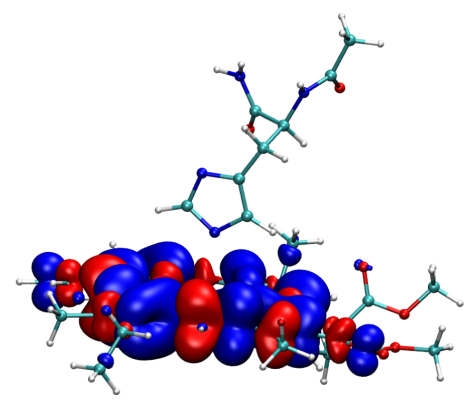

Figure 2: KS-DFT spin densities (isovalue \pm 0.001 a.u.) for the $\left.\left[\mathrm{tP}_{\mathrm{D} 1} \cdots \mathrm{His}\right]\right]^{\bullet}$ model. Red and blue colors show the negative and positive spin-density regions, respectively.

found in Tabs. S1 and S2 of the SI. In these calculations, the initial charge-localized states $\Phi_{1}$ and $\Phi_{2}$ are chosen such that $\Phi_{1} \equiv\left|(\mathrm{t}) \mathrm{P}_{\mathrm{D} 1}{ }^{+\bullet} \ldots \mathrm{HisH}\right\rangle$ and $\Phi_{2} \equiv\left|(\mathrm{t}) \mathrm{P}_{\mathrm{D} 1} \ldots \mathrm{HisH}^{+\bullet}\right\rangle$. As one can see from Tabs. S1 and S2, the calculated diabatic energy levels $H_{11}^{\prime}$ and $H_{22}^{\prime}$ are separated by about $2 \mathrm{eV}$ in all cases. These energy gaps are about $2-3$ orders of magnitude larger than the corresponding electronic couplings $|V|$. Therefore, the charge-localized states $\Phi_{1}$ and $\Phi_{2}$ do not significantly couple and the resulting spindensity distributions are almost completely localized. The calculated electronic ground 
state $\Psi_{0} \approx a_{0}\left|\mathrm{tP}_{\mathrm{D} 1}^{+\bullet} \ldots \mathrm{HisH}\right\rangle$ implies that the spin density is localized at the $\mathrm{tP}_{\mathrm{D} 1}$ molecule, while most of the spin density is localized at HisH in the first excited state $\Psi_{1} \approx b_{1}\left|\mathrm{tP}_{\mathrm{D} 1} \ldots \mathrm{HisH}^{+\bullet}\right\rangle$. This situation is similar for the truncated and non-truncated models and does not change with the intermolecular distance $R$ or tilt of the angle $\theta$. Such modifications of the molecular structures lead to only minor differences in the resulting electronic levels $E_{0}$ and $E_{1}$ (below $0.05 \mathrm{eV}$ ) and spin-density ratios (below $2 \%$ ).

A very different situation can be found for the deprotonated molecular complexes $\left[\mathrm{tP}_{\mathrm{D} 1} \cdots \mathrm{His}\right]^{\bullet}$ and $\left[\mathrm{P}_{\mathrm{D} 1} \cdots \mathrm{His}\right]^{\bullet}$. For these models, FDE-diab calculations employing quasi-diabatic states $\Phi_{1} \equiv\left|(\mathrm{t}) \mathrm{P}_{\mathrm{D} 1}{ }^{+\bullet} \cdots \mathrm{His}^{-}\right\rangle$and $\Phi_{2} \equiv\left|(\mathrm{t}) \mathrm{P}_{\mathrm{D} 1} \cdots \mathrm{His} \bullet^{\bullet}\right\rangle$ were carried out. The resulting energy diagrams and spin-density ratios are shown in Fig. 4. As can be seen, the energy differences between adiabatic states $E_{0}$ and $E_{1}$ are considerably reduced compared to the protonated complexes and are equal to 0.056 and $0.012 \mathrm{eV}$ for the non-modified $\left(\Delta R=0 \AA\right.$ and $\left.\Delta \theta=0^{\circ}\right)$ truncated and non-truncated models, respectively. The diabatic energy difference $\left|H_{11}^{\prime}-H_{22}^{\prime}\right|$ is still much larger than the electronic coupling $|V|$ in any case (for values, see Tabs. S3 and S4 in the SI) and, thus, the corresponding quasi-diabatic states $\Phi_{1}$ and $\Phi_{2}$ do not significantly couple. Interestingly, the FDE-diab approach predicts most of spin-density (99\%-100\%) to be localized at the His molecule in the ground electronic state $\Psi_{0}$ and the opposite spin-density distribution in the first excited state $\Psi_{1}$. The ground-state spin-density ratio does not agree with experimental results, where spin is found to be localized mostly at the $\mathrm{P}_{\mathrm{D} 1}$ co-factor. This spin-density localization at His, however, may be explained by rather small energy differences between the electronic states $\Psi_{0}$ and $\Psi_{1}$. These energy gaps could be sensitive to the geometrical parameters, interactions with the nearby protein environment, and dynamical effects such as thermal molecular motion present in experimental measurements. Therefore, larger molecular models, more sophisticated approaches for geometry optimization, and/or sampling different molecular configurations might be required to reproduce experimental results. The latter is known to have a large influence on the en- 

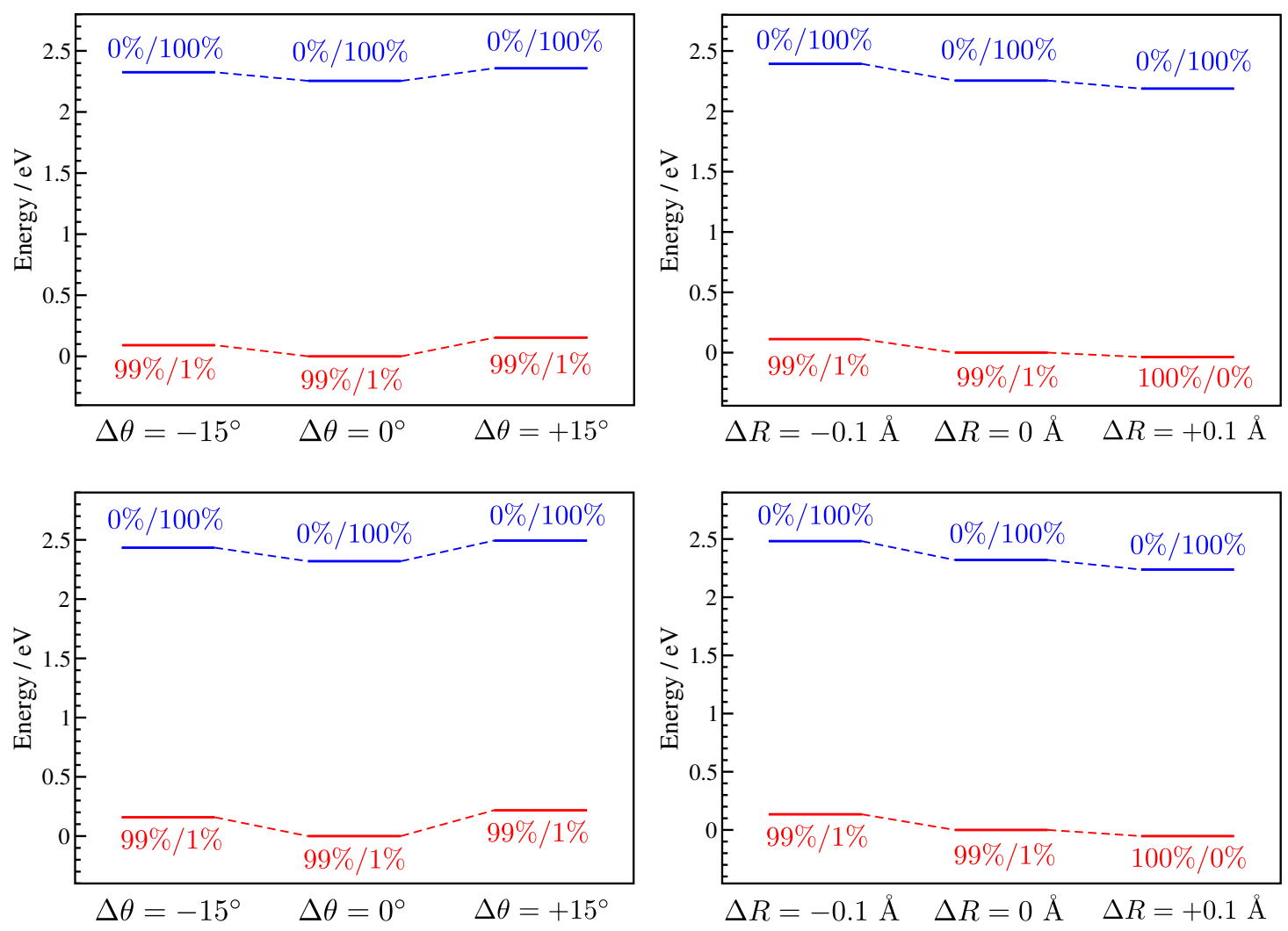

Figure 3: Adiabatic energy levels $E_{0}$ and $E_{1}$ and spin-density ratios (Chl $a$ vs. His) for the $\left[\mathrm{tP}_{\mathrm{D} 1} \cdots \mathrm{HisH}\right]^{+\bullet}(\mathrm{top})$ and $\left[\mathrm{P}_{\mathrm{D} 1} \cdots \mathrm{HisH}\right]^{+\bullet}($ bottom $)$ complex. The diagrams on the left and right sides represent changes with respect to tilt of the angle $\theta$ and modification of the intermolecular distance $R$, respectively. Electronic states with the adiabatic wave function $\Psi$ having the largest contribution from $\Phi_{1}$ are shown in red $(a>b)$, whereas those with the largest contribution from $\Phi_{2}$ are in blue $(a<b)$.

ergetic ordering of MOs [75]. Alternatively, one can argue that the accuracy of FDE-diab is not sufficiently high to correctly reproduce such small energy differences. In fact, this could also be problematic with the standard KS-DFT approach (e.g., see calculations for Mg-porphyrin and Mg-chlorin electronic states in Ref. [27]).

As can be seen from Fig. 4 (right), the energy difference $\Delta E$ grows with the intermolecular 
distance $R$ and the angle $\theta$ for both the truncated and non-truncated model. An increase of the distance $R$ leads to larger energies $E_{0}$ and $E_{1}$, while an increase of the angle $\theta$ lowers the energy level $E_{0}$ and raises $E_{1}$. These modifications of $R$ and $\theta$, however, produce only minor changes $(1 \%-2 \%)$ in spin-density distributions. Larger differences in the spin densities can be observed for negative values of $\Delta R$ and $\Delta \theta$. Thus, in case of the truncated model $\left.\left[\mathrm{tP}_{\mathrm{D} 1} \cdots \mathrm{His}\right]\right]^{\bullet}$ the angle tilt of $\Delta \theta=-15^{\circ}$ increases the value of the electronic coupling $|V|$ to $0.009 \mathrm{eV}$ (see Tab. S3) and simultaneously shrinks the energy gap $\left|H_{11}^{\prime}-H_{22}^{\prime}\right|$ to $0.004 \mathrm{eV}$. This leads to a strong coupling of the quasi-diabatic electronic states $\Phi_{1}$ and $\Phi_{2}$ with a subsequent delocalization of the spin-density. As a result, $40 \%$ and $60 \%$ of the spin-density are localized at $\mathrm{P}_{\mathrm{D} 1}$ in the electronic ground and first excited state, respectively. In addition to the energy diagrams, the dependence of the spin-density distribution on the angle $\theta$ is also demonstrated in Fig. 5. In case of the non-truncated complex, the same tilt $\Delta \theta=-15^{\circ}$ leads to less strongly coupled electronic states, but changes their ordering. Therefore, the ground state localizes $86 \%$ of the spin density on the $\mathrm{P}_{\mathrm{D} 1}$ molecule, whereas the first excited state bears most of the spin density $(86 \%)$ at His. A somewhat similar re-ordering of the electronic states can also be seen for both complexes with intermolecular distances shortened by $\Delta R=-0.1 \AA$. However, this does not considerably increase the coupling between the quasi-diabatic states and, hence, hardly influences the localized character of the resulting spin-density distributions.

Results for binding pocket models (see Tab. S11 in the SI) and hinge models optimized in the pocket and then calculated in vacuum (Tab. S12 in the SI) show essentially identical spin-density distributions as for complexes computed in vacuum. Therefore, we can conclude that the influence of the nearby protein environment on the spin-density distribution in the hinge model from PSII is rather small. This, however, might change if larger parts of the environment are considered.

Analyzing the results from Figs. 3 and 4, a number of conclusions can be drawn. First of 

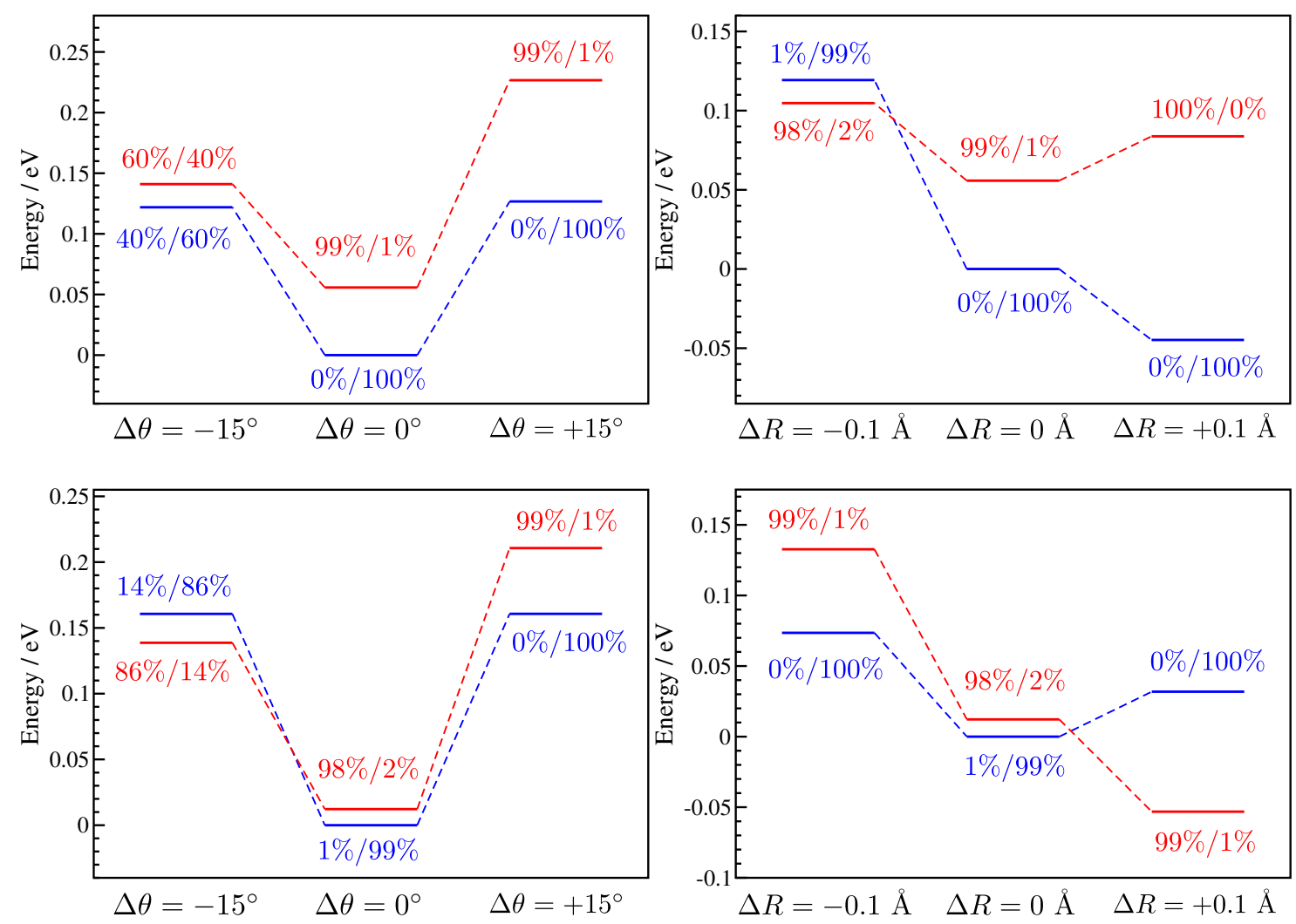

Figure 4: Adiabatic energy levels $E_{0}$ and $E_{1}$ and spin-density ratios (Chl $a$ vs. His) for the $\left[\mathrm{tP}_{\mathrm{D} 1} \cdots \mathrm{His}\right]^{\bullet}(\mathrm{top})$ and $\left[\mathrm{P}_{\mathrm{D} 1} \cdots \mathrm{His}\right]^{\bullet}($ bottom) complex. The diagrams on the left and right side represent changes with respect to tilt of the angle $\theta$ and modification of the intermolecular distance $R$, respectively. Electronic states with the adiabatic wave function $\Psi$ having the largest contribution from $\Phi_{1}$ are shown in red $(a>b)$, whereas those with the largest contribution from $\Phi_{2}$ are in blue $(a<b)$.

all, as can be seen from the spin densities of the non-modified structures $\left(\Delta \theta=0^{\circ}\right.$ and $\Delta R=0 \AA$ ), FDE-diab leads to strongly localized spin-density distributions. These localized spin densities are expected for this type of molecular systems and are in qualitative agreement with experimental measurements. The possibility to obtain such distributions with the rather simple non-hybrid GGA-type PW91 approximation points to the fact 


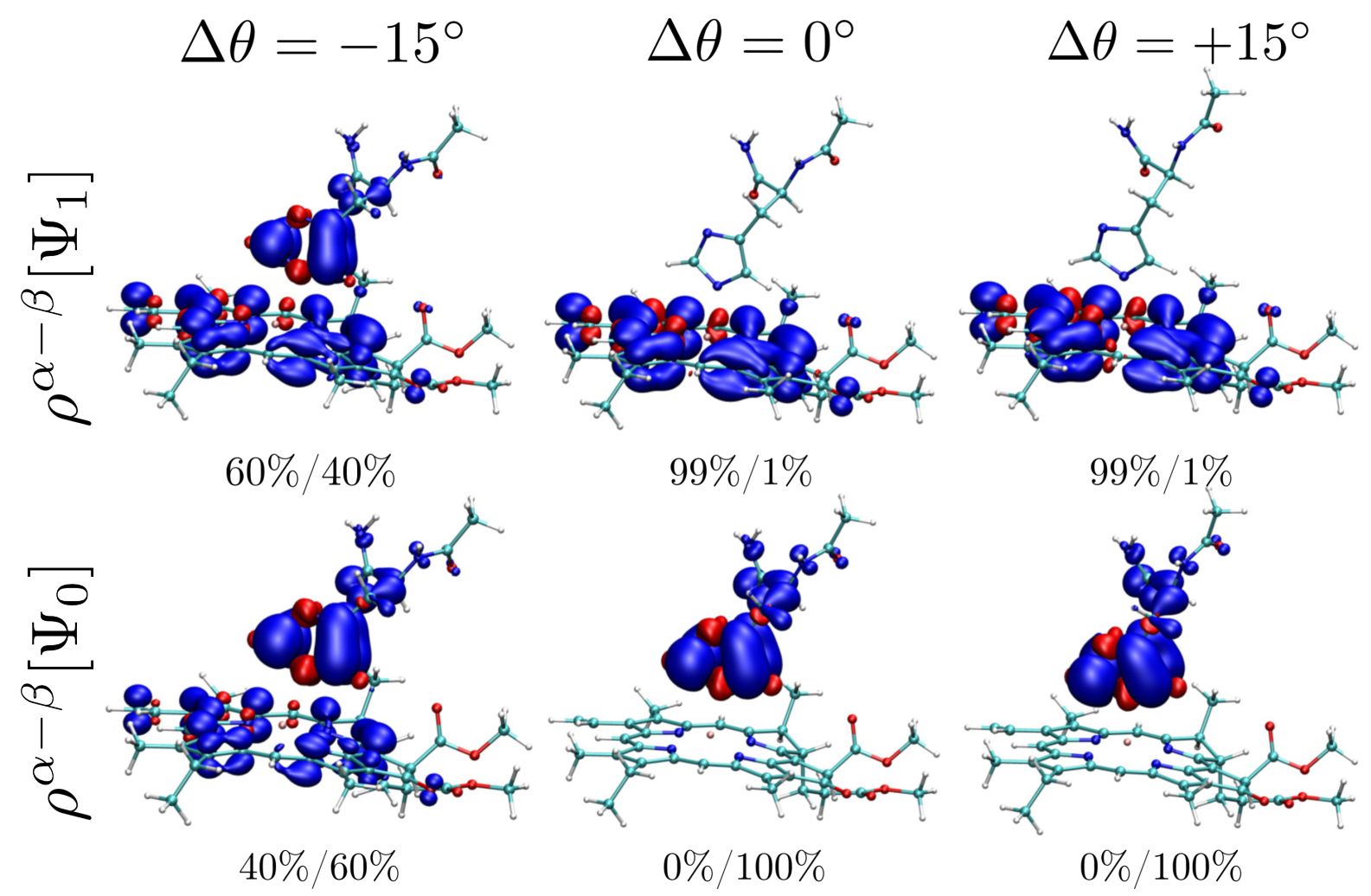

Figure 5: Spin-density distributions (isovalue \pm 0.01 a.u.) and spin population ratios (for $\mathrm{Chl} / \mathrm{His}$; given in \%) for the $\left[\mathrm{tP}_{\mathrm{D} 1} \cdots \mathrm{His}\right]^{\bullet}$ complex and their dependence on the angle $\theta$. Results are presented for the ground $\Psi_{0}$ and first excited $\Psi_{1}$ electronic state. Regions of the positive and negative spin density are shown with blue and red colors, respectively.

that FDE-diab essentially avoids the consequences of the self-interaction-error, as far as intermolecular phenomena are concerned (as has already been highlighted in Refs. [28] and [29]). In addition, FDE-diab does not lead to overestimated areas of negative spin density, in contrast to conventional KS-DFT calculations with hybrid functionals. The FDE-diab spin-density distributions are highly sensitive to modifications of the molecular structure (as can be seen from Fig. 4), while the spin density obtained with, e.g., M06-HF does not show any such dependencies. All of this makes FDE-diab a valuable tool for spin-density calculations with distinct advantages over the standard KS-DFT approach. Secondly, the FDE-diab calculations conducted here partially support the hinge-type model for the RC of PSII. The spin densities obtained for the deprotonated 
models are, indeed, dependent on the angle $\theta$ between the His molecule and the pyrrole ring $D$ of Chl. In line with the interpretation provided in Ref. [3], the angle tilt towards the ring $D$ causes a larger overlap between MOs of Chl $a$ and His (for overlap values, see Tabs. S3 to S4 in the SI) with a subsequent redistribution of the calculated spin density between the two molecules. The ground state spin-density ratio of $86 \% / 14 \%$ obtained for the non-truncated $\left[\mathrm{P}_{\mathrm{D} 1} \cdots \text { His }\right]^{\bullet}$ complex is in excellent agreement with the ENDOR measurements from Ref. [20], which predict $82 \%$ of the spin to be localized at $\mathrm{P}_{\mathrm{D} 1}$. However, based on these findings we cannot discard the possibility that the spin density might be delocalized between all three fragments: His, $\mathrm{P}_{\mathrm{D} 1}$, and $\mathrm{P}_{\mathrm{D} 2}$. Our recent FDE-diab calculations carried out for the inner pair molecules in Ref. [29] showed a considerable electronic coupling between $\mathrm{P}_{\mathrm{D} 1}$ and $\mathrm{P}_{\mathrm{D} 2}$ with the resulting spin-density ratio of about $80 \% / 20 \%$ for these molecules. It should be noted, however, that in FDE-diab calculations reported in Ref. [29], MOs of His were not used for constructions of quasi-diabatic states, thus (by construction) preventing the spin density from delocalizing over the His residue. A decisive conclusion on the spin-density ratio can only be made if all three molecules are used for construction of quasi-diabatic states. This is curently not possible with the available FDE-diab implementation.

\subsection{Reaction Center of Photosystem I}

The molecular complex $\left[\mathrm{P}_{\mathrm{B}} \cdots \mathrm{HisH}\right]$ from PSI is chemically equivalent to those in PSII, whereas $\left[\mathrm{P}_{\mathrm{A}} \cdots \mathrm{HisH}\right]$ features a Chl $a^{\prime}$ molecule instead of Chl $a$. The crystal structure of Synechococcus elongatus [1], however, shows very different mutual orientation of Chl and His molecules in PSI compared to the PSII RC from Thermococcus vulcanus crystal structure [2]. Thus, the intermolecular angle $\theta$ is much smaller and is equal to about 77.7 and 80.2 degrees for $\left[\mathrm{P}_{\mathrm{A}} \cdots \mathrm{HisH}\right]$ and $\left[\mathrm{P}_{\mathrm{B}} \cdots \mathrm{HisH}\right]$, respectively (vs. 93.1 degrees in $\left.\left[\mathrm{P}_{\mathrm{D} 1} \cdots \mathrm{HisH}\right]\right)$. The distance $R$ is slightly larger compared to $\left[\mathrm{P}_{\mathrm{D} 1} \cdots \mathrm{HisH}\right](2.121 \AA)$ and 
is about $2.260 \AA$ in $\left[\mathrm{P}_{\mathrm{A}} \cdots \mathrm{HisH}\right]$ and $2.360 \AA$ in $\left[\mathrm{P}_{\mathrm{B}} \cdots \mathrm{HisH}\right]$. Due to the very similar chemical structures of these complexes, electronic couplings and spin-density distributions are expected to be comparable to those obtained in Sec. 4.1 with exact values defined by an interplay of two factors: i) The negative tilt angle $\theta$ increasing the electronic coupling and spin-density delocalization and ii) enlarged intermolecular distance $R$ causing opposite effects. In addition to the structural differences, the co-factors in PSII and PSI are perturbed differently by the surrounding protein matrices and show distinct extents of asymmetry in experimentally reconstructed spin-density distributions compared to monomeric Chl $a$ in solution [19]. The inner pair co-factors from PSII are known to interact strongly with the nearby protein environment, while the analogous pair in PSI is essentially unperturbed [18,29]. Measured photo-CIDNP MAS NMR signals of PSI were assigned to a single Chl molecule and showed only a slight degree of asymmetry (absence of photo-CIDNP intensities at Chl atoms C-5 and C-20) $[18,76]$. Hovewer, more recent measurements with ${ }^{13} \mathrm{C}$ isotope labels introduced revealed a dimeric nature of the donor in PSI [77]. Similar experiments for PSII reported a highly asymmetrical spin-density distributions over $\mathrm{P}_{\mathrm{D} 1}[3,17,18]$. Substitution of axial His molecules in PSI by other uncharged polar amino acids using site-directed mutagenesis did not lead to significant changes in the spin-density [78] pointing at rather weak interaction between $\mathrm{Chl}$ and His molecules in PSI. The influence of amino acid substitution on ENDOR spectra, however, was much stronger in case of the His molecule coordinated at $\mathrm{P}_{\mathrm{B}}$ than for those at $\mathrm{P}_{\mathrm{A}}$.

The small initial angle $\theta$ in PSI prevents us from further tilting towards ring $D$ as strong steric repulsion effects or group overlaps might occur between the $\mathrm{Chl}$ and His molecules. For this reason, FDE-diab calculations were carried out only for non-modified (with respect to $\theta$ and $R$ ) optimized molecular structures of $\left[\mathrm{P}_{\mathrm{A}} \cdots \mathrm{HisH}\right]$ and $\left[\mathrm{P}_{\mathrm{B}} \cdots \mathrm{HisH}\right]$. The results of these calculations are presented in Tabs. S5 and S6 in the SI. As can be seen from these data, both protonated complexes $\left[\mathrm{tP}_{\mathrm{A}} \cdots \mathrm{HisH}\right]^{+\bullet}$ and $\left[\mathrm{tP}_{\mathrm{B}} \cdots \mathrm{HisH}\right]^{+\bullet}$ show very similar values for diabatic energy differences $\left|H_{11}^{\prime}-H_{22}^{\prime}\right|$ of about 2.6 and $2.2 \mathrm{eV}$, respec- 
tively. The corresponding electronic couplings are slightly larger than those obtained for PSII models, but still much smaller than the energy gaps $\left|H_{11}^{\prime}-H_{22}^{\prime}\right|$. Therefore, coupling of electronic states does not occur and the ground state spin-density distributions stay highly localized at the $\mathrm{tP}_{\mathrm{A}} / \mathrm{tP}_{\mathrm{B}}$ co-factors with a contribution of $99-100 \%$, while about the same amount of the spin density can be found at HisH molecules in the first excited electronic state. Non-truncated models of $\left[\mathrm{P}_{\mathrm{A}} \cdots \mathrm{HisH}\right]^{+\bullet}$ and $\left[\mathrm{P}_{\mathrm{B}} \cdots \mathrm{HisH}\right]^{+\bullet}$ show very similar behavior with unchanged spin-density distributions and only minor changes (a few meV) in $\left|H_{11}^{\prime}-H_{22}^{\prime}\right|$ and $|V|$. The truncation of hydrocarbon tails, therefore, does not strongly affect the results. Inclusion of the nearby protein environment only slightly changes the values of excitation energies and electronic couplings and leads to essentially unchanged spin-density distributions (see Tab. S11 in the SI). A similar distribution is observed for the model optimized in the binding pocket but calculated in vacuum (Tab. S12 in the SI).

As expected, deprotonation at the $\pi$ - $\mathrm{N}$ position of the HisH molecules results in much smaller energy gaps $\left|H_{11}^{\prime}-H_{22}^{\prime}\right|$ (see Tab. S6 in the SI). This also leads to a coupling of the diabatic electronic states $\Phi_{1}$ and $\Phi_{2}$ in both $\left[\mathrm{tP}_{\mathrm{A}} \cdots \mathrm{His}\right]^{\bullet}$ and $\left[\mathrm{tP}_{\mathrm{B}} \cdots \mathrm{His}\right]^{\bullet}$ complexes. However, the values of $|V|$ are about six times smaller than the corresponding energy differences $\left|H_{11}^{\prime}-H_{22}^{\prime}\right|$. Therefore, only about $3-4 \%$ of the spin-density is localized at $\mathrm{His}^{-}$in the electronic ground state (and 96-97\% in the first excited state). These results are quite different from those obtained for tilted hinge models of PSII, where much larger delocalization (up to $40 \% / 60 \%$ for $\theta \approx 78^{\circ}$ ) was observed. This small delocalization is rather surprising considering the angles $\theta$ in both PSI molecular models. This small delocalization degree may probably be explained by the larger intermolecular distances $R$ in the corresponding PSI complexes. Again, non-truncated models $\left[\mathrm{P}_{\mathrm{A}} \cdots \mathrm{His}\right]^{\bullet}$ and $\left[\mathrm{P}_{\mathrm{B}} \cdots \mathrm{His}\right]^{\bullet}$ show very similar results compared to their truncated counterparts. It is interesting to note that deprotonated models comprising the $\mathrm{P}_{\mathrm{A}}$ co-factor show about three times larger values of both electronic couplings $|V|$ and energy differences $\left|H_{11}^{\prime}-H_{22}^{\prime}\right|$ than those with 
$\mathrm{P}_{\mathrm{B}}$. This, however, does not influence the resulting spin-density distributions. Another aspect that should be noted is the magnitude of the energy gap $\left|H_{11}^{\prime}-H_{22}^{\prime}\right|$, which is much larger than the gaps calculated for the deprotonated hinge models of PSII. In the latter case, the energy levels $H_{11}^{\prime}$ and $H_{22}^{\prime}$ were nearly degenerate and, therefore, changed their ordering with modifications in the mutual orientation of fragments (see Sec. 4.1). This does not occur in deprotonated models of PSI, where the initial ordering of the electronic ground and first excited state remains unchanged. The interaction with the surrounding protein slightly changes the spin-density distribution resulting in the ratio of $94 \% / 6 \%$ (see Tab. S11 in the SI). Therefore, the obtained results for both protonated and deprotonated complexes in PSI show only a minor degree of the spin-density delocalization between $\mathrm{Chl}$ and His molecules. This agrees well with experimental photo-CIDNP NMR

studies $[3,18,76]$, where all spectroscopic signals were attributed to either a single Chl $a$ molecule $[76]$ or the $\left[\mathrm{P}_{\mathrm{A}} \cdots \mathrm{P}_{\mathrm{B}}\right]$ dimer $[3,77]$. Unfortunately, this small degree of delocalization in the calculated complexes prevents us from drawing an unambiguous conclusion about the protonation state of the axial His molecules in PSI.

\subsection{Bacterial Reaction Center}

Similar to the case of PSII [3], photo-CIDNP MAS NMR signals originating from nitrogen atoms of axial His molecules were tentatively assigned in bacterial RC of ${ }^{15} \mathrm{~N}$ labeled Rhodobacter sphaeroides R26 [79,80]. The presence of these signals, however, was explained by indirect spin diffusion or rotational resonance processes. The authors of Ref. [81] also discussed the possiblity to assign some of the measured ${ }^{13} \mathrm{C}$ photo-CIDNP signals in the Rhodobacter sphaeroides wild type (WT) to either axial histidine atoms or bacteriopheophytin (BPhe). In this case, it was not possible to draw a definite conclusion without further experimental data with selective isotope labeling or oriented samples. More recent photo-CIDNP MAS NMR measurements of ${ }^{15} \mathrm{~N}$-labeled Rhodobacter 
sphaeroides WT reported in Ref. [82] showed that all signals originate from the special pair co-factors and BPhe. Nowadays it is generally accepted that the axial His molecule in bacterial RC does not carry any spin-density contributions. KS-DFT calculations presented in Ref. [83] showed a minor influence of axial ligation to the spin density of a single BChl a molecule. According to the results in Ref. [80], axial His molecules are protonated at $\pi$-N positions. All four axial His molecules coordinated at inner and accessory BChls were also found to be protonated in Ref. [84] based on results of cross-polarization-magicangle spinning (CP-MAS) ${ }^{15} \mathrm{~N}$ for Rhodobacter sphaeroides $\mathrm{R} 26$. The properties of one of the His residues were, however, reported to be different from the other three due to formation of a strong $\mathrm{X}-\mathrm{H}-\pi-\mathrm{N}$ hydrogen bond. Based on these experimental findings, we assume in the following that i) the $\mathrm{D}_{\mathrm{A}}{ }^{+\bullet}$ cation radical is coordinated by a protonated His molecule and ii) the spin density is fully localized at the $\mathrm{D}_{\mathrm{A}}^{+} \cdot \boldsymbol{c o}$-factor (when $\mathrm{D}_{\mathrm{B}}$ is not included into the model).

In contrast to the PSI models, the relative orientation of $\mathrm{BChl} a$ and $\mathrm{HisH}$ in $\left[\mathrm{D}_{\mathrm{A}} \cdots \mathrm{HisH}\right]$ is much closer to the case of the hinge model of PSII. The crystal structure shows that the angle $\theta$ is equal to $95.6^{\circ}$, whereas the intermolecular distance $R$ is of about $2.225 \AA$ (vs. $\theta=93.1^{\circ}$ and $R=2.121 \AA$ in PSII). Therefore, strong steric repulsion does not occur for tilted structures and a number of modified molecular models (similar to those in Sec. 4.1) can be created to study the spin-density delocalization. Detailed results of FDE-diab calculations for such models are presented in Tabs. S7-S10 in the SI. The constructed charge-localized states used in these calculations are $\Phi_{1} \equiv\left|(\mathrm{t}) \mathrm{D}_{\mathrm{A}}{ }^{+\bullet} \ldots \mathrm{HisH}\right\rangle$ and $\Phi_{2} \equiv\left|(\mathrm{t}) \mathrm{D}_{\mathrm{A}} \ldots \mathrm{HisH}^{+\bullet}\right\rangle$ for protonated models and $\Phi_{1} \equiv\left|(\mathrm{t}) \mathrm{D}_{\mathrm{A}}{ }^{+\bullet} \ldots \mathrm{His}^{-}\right\rangle$and $\Phi_{2} \equiv$ $\left|(t) D_{\mathrm{A}} \ldots \mathrm{His}^{\bullet}\right\rangle$ for their deprotonated counterparts.

As can be seen from Tabs. S7 and S8, similar to the previously shown results for PSII and PSI models, coupling of electronic states does not occur in the protonated complexes $\left[\mathrm{tD}_{\mathrm{A}} \cdots \mathrm{HisH}\right]^{+\bullet}$ and $\left[\mathrm{D}_{\mathrm{A}} \cdots \mathrm{HisH}\right]^{+\bullet}$. The diabatic energy gap $\left|H_{11}^{\prime}-H_{22}^{\prime}\right|$ is equal to about 
$2.0 \mathrm{eV}$ for both truncated and non-truncated models and for all intermolecular angles $\theta$ and distances $R$ presented, while the value of $|V|$ is about two orders of magnitude smaller than $\left|H_{11}^{\prime}-H_{22}^{\prime}\right|$. As a result, the spin density is completely $(99-100 \%)$ localized at the $\mathrm{D}_{\mathrm{A}}$ co-factor in the electronic ground state and at the HisH molecule in the first excited state. Inclusion of the nearby protein environment does not change the spin-density distribution in the hinge-type model in the bacterial RC (see Tab. S11 in the SI).

Energy level diagrams for the deprotonated complexes $\left[\mathrm{tD}_{\mathrm{A}} \cdots \mathrm{His}\right]^{\bullet}$ and $\left[\mathrm{D}_{\mathrm{A}} \cdots \mathrm{His}\right]^{\bullet}$ are shown in Fig. 6, while detailed lists of calculated values are presented in Tabs. S9 and S10 in the SI. As can be seen from Fig. 6, the ground state spin-density distribution is completely localized at His ${ }^{\bullet}$ in the molecular models. Modification of the angle $\theta$ by $\Delta \theta=+15^{\circ}$ slightly increases the diabatic energies $H_{11}^{\prime}$ and $H_{22}^{\prime}$ and the corresponding electronic couplings $|V|$ (to $30 \mathrm{meV}$ ). Because the energy gap $\left|H_{11}^{\prime}-H_{22}^{\prime}\right|$ is still about ten times larger than $|V|$, only minor spin-density delocalization occur in the electronic ground $(1 \% / 99 \%)$ and first excited $(98 \% / 2 \%)$ state. Much larger effects are observed in case of negative modification of the angle $\theta$. For $\Delta \theta=-15^{\circ}$, the electronic coupling $|V|$ increases from 0 to $142 \mathrm{meV}$ for both complexes, while the gap $\left|H_{11}^{\prime}-H_{22}^{\prime}\right|$ becomes smaller and reaches 278 and $280 \mathrm{meV}$ for $\left[\mathrm{tD}_{\mathrm{A}} \cdots \mathrm{His}\right]^{\bullet}$ and $\left[\mathrm{D}_{\mathrm{A}} \cdots \mathrm{His}\right]^{\bullet}$, respectively. This leads to a strong coupling of the corresponding diabatic electronic states and induces delocalization of the spin-density. For both molecular models, the resulting spin-density ratios are $16 \% / 84 \%$ and $85 \% / 15 \%$ in the electronic ground and first excited state, respectively. The value of $H_{11}^{\prime}-H_{22}^{\prime}$ varies in the range of $280-350 \mathrm{meV}$ for all angles $\theta$ calculated and does not change the sign. Therefore, the order of diabatic electronic states remains the same. As can be seen from Tab. S11 in the SI, accounting for the interactions with the surrounding protein environment changes the energy-level ordering, thus resulting in the anticipated ground electronic state spin-density ratios of $99 \% / 1 \%$ in favor of $\mathrm{D}_{\mathrm{A}}$ for both protonated and deprotonated models. 

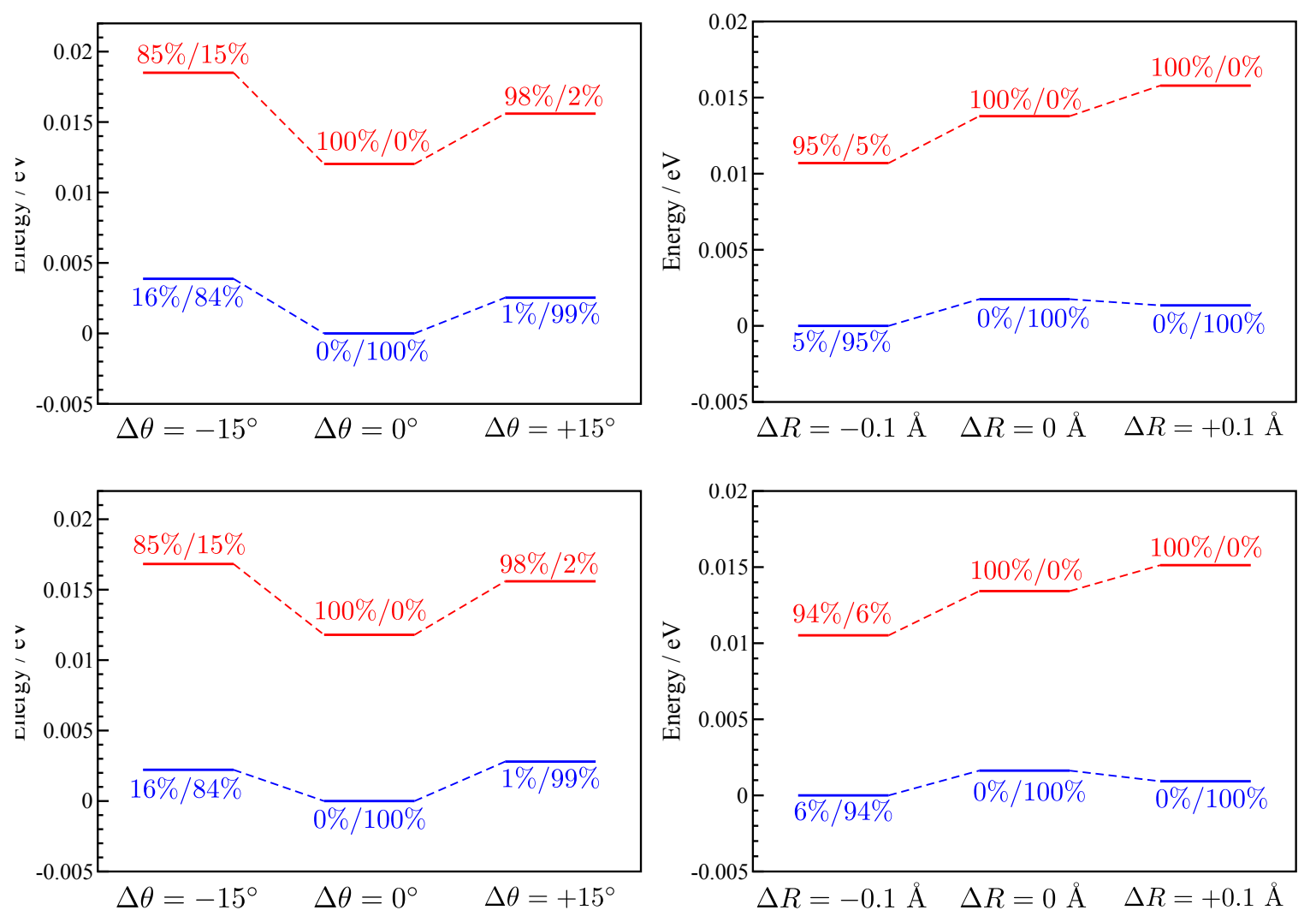

Figure 6: Adiabatic energy levels $E_{0}$ and $E_{1}$ and spin-density ratios (BChl $a$ vs. His) for the $\left[\mathrm{tD}_{\mathrm{A}} \cdots \mathrm{His}\right]^{\bullet}(\mathrm{top})$ and $\left[\mathrm{D}_{\mathrm{A}} \cdots \mathrm{His}\right]^{\bullet}$ (bottom) complex. The diagrams on the left and right sides represent changes with respect to tilt of the angle $\theta$ and modification of the intermolecular distance $R$, respectively. Electronic states with the adiabatic wave function $\Psi$ having the largest contribution from $\Phi_{1}$ are shown in red $(a>b)$, whereas those with the largest contribution from $\Phi_{2}$ are in blue $(a<b)$.

Interestingly, enlargement of the intermolecular distance $R$ by $+0.1 \AA$ in deprotonated complexes leads to slightly larger (by $5 \mathrm{meV}$ ) electronic coupling values (see Tabs. S9 and S10). However, this also increases energy gaps $\left|H_{11}^{\prime}-H_{22}^{\prime}\right|$. Therefore, coupling of diabatic electronic states does not occur and spin-density distributions remain unchanged. Much larger changes in the electronic couplings can be seen for $\Delta R=-0.1 \AA$. In this case, $|V|$ increases by $63-64 \mathrm{meV}$ with a simultaneous change in the energy gaps of -65 
meV. This leads to a weak coupling of electronic states and a small (5-6\%) spin-density delocalization in the ground and first excited states of the $\left[\mathrm{tD}_{\mathrm{A}} \cdots \mathrm{His}\right]^{\bullet}$ and $\left[\mathrm{D}_{\mathrm{A}} \cdots \mathrm{His}\right]^{\bullet}$ molecular complexes. Similar to the case of PSI, the long hydrocarbon tail R of the BChl $a$ molecule does not affect the calculated parameters. Thus, only the hinge model in PSII features nearly degenerate adiabatic electronic states and shows considerable changes in the resulting electronic couplings and spin-density distribution upon the truncation.

In contrast to the results obtained for the PSI model, calculated spin densities for deprotonated models from bacterial RC show a noticeable dependency on the modifications in the relative orientation of molecules composing the hinge-like model. The degree of spin delocalization differs by up to $16 \%$ upon axial His tilting and up to $6 \%$ upon changes in the intermolecular distance between BChl $a$ and His. The experimental results indicate completely localized spin-density distributions in the bacterial RC (see Refs. [79-81]), which are in good agreement with those calculated for protonated complexes. Therefore, it can be concluded that the axial His molecules in the bacterial RC are most likely protonated.

\section{Conclusions}

In this work, we investigated spin-density distrubutions and electronic couplings in RCs of photosynthetic systems using the recently developed FDE-diab approach [28]. This technique is known to be highly efficient for calculations of large open-shell molecular systems [29] and to effectively avoid the spin-density overdelocalization problem $[25,26]$ characteristic to standard KS-DFT. Its high sensitivity to changes in molecular structures allowed us to validate a number of hinge-like molecular models for primary electron donors in RCs.

Our results demonstrate a very high sensitivity of FDE-diab to the protonation state of the axial His molecule. Thus, protonated radical cation complexes of (B)Chl $a$ and His from 
PSII, PSI, and bacterial RC featured energetically well separated (by about 2.0-2.6 eV) and uncoupled electronic states. The resulting spin-density distributions were, therefore, almost fully localized at (B)Chl $a$ for all intermolecular angles and distances considered. For these models, inclusion of the nearby protein environment did not considerably affect the spin distributions. In the hinge model from PSII, deprotonation of the His molecule at the $\pi$-N position drastically decreased the electronic levels separation and led to couplings of the quasi-diabatic states at acute angles $\theta$ with a subsequent shifts of the spin density towards His. This effect of spin-density re-distribution was previosly predicted on the basis of experimental results in Ref. [3]. Our calculations, therefore, partially support the proposed explanation and show that the tilt of the angle $\theta$ can, indeed, cause a change in the spin density of the complex. Calculations for the truncated hinge model from PSII showed a wrong spin-density localization with $60 \%$ being localized at His in the ground state at $\Delta \theta=-15$ degrees. This was explained by an inverted ordering of the nearly degenerate ground and excited electronic states of the complex. The non-truncated model demonstrated a correct spin-density localization at Chl $a(86 \%)$ and agreed well with ENDOR measurements [20] predicting $82 \%$ of spin density at Chl $a$. Contrary to the results reported in Ref. [3], we did not observe a considerable shift of the spin density within Chl $a$ under the angle tilt. Therefore, the reported strong asymmetry in the spin-density pattern of Chl $a$ was not observed in calculated FDE-diab spin densities. Noticeable electronic couplings for inner pair molecules [29] and for $\mathrm{P}_{\mathrm{D} 1}$ and His (at acute angles between them) point out at the possible spin-density delocalization between all three molecules with the largest contribution on $\mathrm{P}_{\mathrm{D} 1}$. Again, inclusion of the nearby protein environment does not strongly affect the resulting distributions.

In case of the hinge-type model from PSI, deprotonation of His at the $\pi$-N possition did not lead to a considerable change in the spin density. This was rather surprising taking into account an acute angle between His and $\mathrm{P}_{\mathrm{A}}$ in the original crystal structure. It can be explained by a large distance and weak interactions between these molecules. To the 
best of our knowledge, there is no experimental evidence available about spin density being present at the axial His molecules in PSI. Therefore, our results do not contradict any experimetal measurements. However, they also do not provide us with any hints on the true protonation state of the axial His molecule in PSI.

Similar to the case of PSII, deprotonation at the axial His molecule of bacterial RC led to noticeable delocalizaton of the spin density for acute angles between His and $\mathrm{D}_{\mathrm{A}}$. Thus, spin-density ratios of isolated truncated and non-truncated hinge models at $\Delta \theta=-15$ degree were both equal to $16 \% / 84 \%$ in favor of His. It is, however, accepted that the axial His molecule in the bacterial RC does not have spin-density contributions. Therefore, taking into account the large degrees of spin-density localizations at the His residue for deprotonated models calculated with FDE-diab, one might conclude that the axial His from the bacterial RC most likely appears to be protonated. This agrees well with available experimental results $[80,84]$.

Despite a clear success of the FDE-diab approach in predicting qualitatively correct spindensity distributions, this methodology as well as the computational protocol used in this work still leave room for improvements. Unambiguous predictions of spin-density delocalizations over multiple subsystems are not possible within the described above twostate model. These calculations would require a generalization of the FDE-diab towards multiple quasi-diabatic states. Another aspect to be mentioned is the use of rigid molecular structures, which does not account for dynamical effects such as thermal molecular motion. These effects are known to have a large influence on MO energies [75].

\section{Acknowledgments}

D.G.A. acknowledges funding from the European Union's Horizon 2020 research and innovation programme under the Marie Skłodowska-Curie grant agreement no. 835776. 
J.M. and J.N. acknowledge funding by the Deutsche Forschungsgemeinschaft through the combined projects MA 4972/2-1 and NE 912/3-1. 


\section{References}

[1] P. Jordan, P. Fromme, H. T. Witt, O. Klukas, W. Saenger, N. Krauss. Threedimensional structure of cyanobacterial photosystem I at $2.5 \AA$ resolution. Nature, 411 (2001) 909-917.

[2] Y. Umena, K. Kawakami, J.-R. Shen, N. Kamiya. Crystal structure of oxygenevolving photosystem II at a resolution of $1.9 \AA$. Nature, 473 (2011) 55-61.

[3] A. Diller, E. Roy, P. Gast, H. J. van Gorkom, H. J. M. de Groot, C. Glaubitz, G. Jeschke, J. Matysik, A. Alia. ${ }^{15} \mathrm{~N}$ photochemically induced dynamic nuclear polarization magic-angle spinning NMR analysis of the electron donor of photosystem II. PNAS, 104 (2007) 12767-12771.

[4] M. Najdanova, G. J. Janssen, H. J. M. de Groot, J. Matysik, A.Alia. Analysis of electron donors in photosystems in oxygenic photosynthesis by photo-CIDNP MAS NMR. J. Photochem. Photobiol. B, 152 (2015) 261-271.

[5] B. A. Diner, F. Rappaport. Structure, Dynamics, and Energetics of the Primary Photochemistry of Photosystem II of Oxygenic Photosynthesis. Annu. Rev. Plant Biol., 53(1) (2002) 551-580.

[6] T. Cardona, A. Sedoud, N. Cox, A. W. Rutherford. Charge separation in photosystem ii: A comparative and evolutionary overview. BBA - Bioenergetics, 1817(1) (2012) $26-43$.

[7] H.-G. Duan, V. I. Prokhorenko, E. Wientjes, R. Croce, M. Thorwart, D. R. J. Miller. Primary Charge Separation in the Photosystem II Reaction Center Revealed by a Global Analysis of the Two-dimensional Electronic Spectra. Scientific Reports, 7(1) (2017) 12347. 
[8] O. G. Poluektov, S. V. Paschenko, L. M. Utschig, K. V. Lakshmi, M. C. Thurnauer. Bidirectional Electron Transfer in Photosystem I: Direct Evidence from HighFrequency Time-Resolved EPR Spectroscopy. J. Am. Chem. Soc., 127(34) (2005) $11910-1911$.

[9] S. Santabarbara, I. Kuprov, P. J. Hore, A. Casal, P. Heathcote, M. C. W. Evans. Analysis of the Spin-Polarized Electron Spin Echo of the [P700+A1-] Radical Pair of Photosystem I Indicates That Both Reaction Center Subunits Are Competent in Electron Transfer in Cyanobacteria, Green Algae, and Higher Plants. Biochemistry, 45(23) (2006) $7389-7403$.

[10] G. J. Janssen, E. Daviso, M. van Son, H. J. M. de Groot, A. Alia, J. Matysik. Observation of the solid-state photo-CIDNP effect in entire cells of cyanobacteria Synechocystis. Photosynth. Res., 104 (2010) 275-282.

[11] G. J. Janssen, P. Bielytskyi, D. G. Artiukhin, J. Neugebauer, H. J. M. de Groot, J. Matysik, A. Alia. Photochemically induced dynamic nuclear polarization NMR on photosystem II: donor cofactor observed in entire plant. Sci. Rep., 8 (2018) 17853.

[12] E. A. M. Schulten, J. Matysik, A. Alia, S. Kiihne, J. Raap, J. Lugtenburg, P. Gast, A. J. Hoff, H. J. M. de Groot. ${ }^{13} \mathrm{C}$ MAS NMR and Photo-CIDNP Reveal a Pronounced Asymmetry in the Electronic Ground State of the Special Pair of Rhodobacter sphaeroides Reaction Centers. Biochemistry, 41 (2002) 8708-8717.

[13] S. Prakash, A. Alia, P. Gast, G. Jeschke, H. J. M. de Groot, J. Matysik. Photochemically induced dynamic nuclear polarisation in entire bacterial photosynthetic units observed by ${ }^{13} \mathrm{C}$ magic-angle spinning NMR. J. Mol. Struct., 661 (2003) 625-633.

[14] A. Diller, S. Prakash, A. Alia, P. Gast, J. Matysik, G. Jeschke. Signals in SolidState Photochemically Induced Dynamic Nuclear Polarization Recover Faster Than 
Signals Obtained with the Longitudinal Relaxation Time. J. Phys. Chem. B, 111 (2007) 10606-10614.

[15] E. Daviso, S. Prakash, A. Alia, P. Gast, J. Neugebauer, G. Jeschke, J. Matysik. The electronic structure of the primary electron donor of reaction centers of purple bacteria at atomic resolution as observed by photo-CIDNP ${ }^{13} \mathrm{C}$ NMR. PNAS, 106 (2009) 22281-22286.

[16] S. S. Thamarath, B. E. Bode, S. Prakash, K. B. S. S. Gupta, A. Alia, G. Jeschke, J. Matysik. Electron Spin Density Distribution in the Special Pair Triplet of Rhodobacter sphaeroides R26 Revealed by Magnetic Field Dependence of the SolidState Photo-CIDNP Effect. J. Am. Chem. Soc., 134 (2012) 5921-5930.

[17] J. Matysik, A. Alia, P. Gast, H. J. van Gorkom, A. J. Hoff, H. J. M. de Groot. Photochemically induced nuclear spin polarization in reaction centers of photosystem II observed by ${ }^{13} \mathrm{C}$-solid-state NMR reveals a strongly asymmetric electronic structure of the $\mathrm{P}_{680}^{+}$primary donor chlorophyll. PNAS, 97 (2000) 9865-9870.

[18] A. Diller, A. Alia, E. Roy, P. Gast, H. J. van Gorkom, J. Zaanen, H. J. M. de Groot, C. Glaubitz, J. Matysik. Photo-CIDNP solid-state NMR on Photosystems I and II: what makes P680 special? Photosynth. Res., 84 (2005) 303-308.

[19] H. Käß, E. Bittersmann-Weidlich, L.-E. Andréasson, B. Bönigk, W. Lubitz. ENDOR and ESEEM of the $15 \mathrm{~N}$ labelled radical cations of chlorophyll a and the primary donor P700 in photosystem I. Chem. Phys., 194 (1995) 419-432.

[20] S. E. J. Rigby, J. H. A. Nugent, P. J. O’Malley. ENDOR and Special Triple Resonance Studies of Chlorophyll Cation Radicals in Photosystem 2. Biochemistry, 33 (1994) $10043-10050$.

[21] T. A. Wesołowski, A. Warshel. Frozen density functional approach for ab initio calculations of solvated molecules. J. Phys. Chem., 97 (1993) 8050-8053. 
[22] P. Mori-Sánchez, A. J. Cohen, W. Yang. Many-electron self-interaction error in approximate density functionals. J. Chem. Phys., 125 (2006) 201102.

[23] J. Gräfenstein, E. Kraka, D. Cremer. Effect of the self-interaction error for threeelectron bonds: On the development of new exchange-correlation functionals. Phys. Chem. Chem. Phys., 6 (2004) 1096-1112.

[24] A. J. Cohen, P. Mori-Sánchez, W. Yang. Development of exchange-correlation functionals with minimal many-electron self-interaction error. J. Chem. Phys., 126 (2007) 191109.

[25] A. J. Cohen, P. Mori-Sánchez, W. Yang. Insights into Current Limitations of Density Functional Theory. Science, 321 (2008) 792-794.

[26] A. Solovyeva, M. Pavanello, J. Neugebauer. Spin densities from subsystem densityfunctional theory: Assessment and application to a photosynthetic reaction center complex model. J. Chem. Phys., 136 (2012) 194104.

[27] D. G. Artiukhin, C. J. Stein, M. Reiher, J. Neugebauer. Quantum Chemical Spin Densities for Radical Cations of Photosynthetic Pigment Models. Photochem. Photobiol., 93 (2017) 815-833.

[28] D. G. Artiukhin, J. Neugebauer. Frozen-density embedding as a quasi-diabatization tool: Charge-localized states for spin-density calculations. J. Chem. Phys., 148 (2018) 214104.

[29] D. G. Artiukhin, P. Eschenbach, J. Neugebauer. Computational investigation of the spin-density asymmetry in photosynthetic reaction center models from first principles. J. Phys. Chem. B, 124 (2020) 4873-4888.

[30] M. Pavanello, J. Neugebauer. Modelling charge transfer reactions with the frozen density embedding formalism. J. Chem. Phys., 135 (2011) 234103. 
[31] M. Pavanello, T. Van Voorhis, L. Visscher, J. Neugebauer. An accurate and linearscaling method for calculating charge-transfer excitation energies and diabatic couplings. J. Chem. Phys., 138 (2013) 054101.

[32] A. Solovyeva, M. Pavanello, J. Neugebauer. Describing long-range charge-separation processes with subsystem density-functional theory. J. Chem. Phys., 140 (2014) 164103.

[33] P. Ramos, M. Papadakis, M. Pavanello. Performance of Frozen Density Embedding for Modeling Hole Transfer Reactions. J. Phys. Chem. B, 119 (2015) 7541-7557.

[34] T.A. Wesołowski. Computational Chemistry: Reviews of Current Trends, Volume 10, Chapter One-Electron Equations for Embedded Electron Density: Challenge for Theory and Practical Payoffs in Multi-Level Modelling of Complex Polyatomic Systems, p. 1-82. World Scientific, 2006.

[35] A. Migliore, S. Corni, D. Versano, M. L. Klein, R. Di Felice. First Principles Effective Electronic Couplings for Hole Transfer in Natural and Size-Expanded DNA. J. Phys. Chem. B, 113 (2009) 9402-9415.

[36] A. Migliore. Nonorthogonality Problem and Effective Electronic Coupling Calculation: Application to Charge Transfer in $\pi$-Stacks Relevant to Biochemistry and Molecular Electronics. J. Chem. Theory Comput., 7 (2011) 1712-1725.

[37] S. Efrima, M. Bixon. Vibrational effects in outer-sphere electron-transfer reactions in polar media. Chem. Phys., 13 (1976) 447-460.

[38] M. D. Newton. Quantum chemical probes of electron-transfer kinetics: the nature of donor-acceptor interactions. Chem. Rev., 91 (1991) 767-792.

[39] A. Camara-Artigas, D. Brune, J. P. Allen. Interactions between lipids and bacterial reaction centers determined by protein crystallography. PNAS, 99 (2002) 1105511060. 
[40] J. M. Word, S. C. Lovell, J. S. Richardson, D. C. Richardson. Asparagine and Glutamine: Using Hydrogen Atom Contacts in the Choice of Side-chain Amide Orientation. J. Mol. Biol., 285 (1999) 1735-1747.

[41] A.D. MacKerell, M. Feig, C. L. Brooks. Extending the treatment of backbone energetics in protein force fields: Limitations of gasphase quantum mechanics in reproducing protein conformational distributions in molecular dynamics simulations. J. Comput. Chem., 25 (2004) 1400-1415.

[42] A. D. MacKerell, D. Bashford, M. Bellott, R. L. Dunbrack, J. D. Evanseck, M. J. Field, S. Fischer, J. Gao, H. Guo, S. Ha, D. Joseph-McCarthy, L. Kuchnir, K. Kuczera, F. T. K. Lau, C. Mattos, S. Michnick, T. Ngo, D. T. Nguyen, B. Prodhom, W. E. Reiher, B. Roux, M. Schlenkrich, J. C. Smith, R. Stote, J. Straub, M. Watanabe, J. Wiórkiewicz-Kuczera, D. Yin, M. Karplus. All-atom empirical potential for molecular modeling and dynamics studies of proteins. The Journal of Physical Chemistry B, 102 (1998) 3586-3616.

[43] F. H. Allen, O. Kennard, D. G. Watson, L. Brammer, A. G. Orpen, R. Taylor. Tables of bond lengths determined by X-ray and neutron diffraction. Part 1. Bond lengths in organic compounds. J. Chem. Soc., Perkin Trans. 2, 1987) S1.

[44] F. Neese. The ORCA program system. Comput. Mol. Sci., 2 (2012) 73-78.

[45] A. D. Becke. Density-functional exchange-energy approximation with correct asymptotic behavior. Phys. Rev. A, 38(6) (1988) 3098-3100.

[46] J. P. Perdew. Density-functional approximation for the correlation energy of the inhomogeneous electron gas. Phys. Rev. B, 33 (1986) 8822-8824.

[47] A. Schäfer, H. Horn, R. Ahlrichs. Fully optimized contracted Gaussian basis sets for atoms Li to Kr. J. Chem. Phys., 97 (1992) 2571-2577. 
[48] A. Schäfer, C. Huber, R. Ahlrichs. Fully optimized contracted Gaussian basis sets of triple zeta valence quality for atoms Li to Kr. J. Chem. Phys., 100 (1994) 5829.

[49] S. Grimme, J. Antony, S. Ehrlich, H. Krieg. A consistent and accurate ab initio parametrization of density functional dispersion correction (DFT-D) for the 94 elements H-Pu. J. Chem. Phys., 132 (2010) 154104.

[50] S. Grimme, S. Ehrlich, L. Goerigk. Effect of the damping function in dispersion corrected density functional theory. J. Comput. Chem., 32 (2011) 1456-1465.

[51] Amsterdam density functional program, Theoretical Chemistry, Vrije Universiteit, Amsterdam, URL: http://www.scm.com, access date: 13 January 2018.

[52] M. Gaus, A. Goez, M. Elstner. Parametrization and Benchmark of DFTB3 for Organic Molecules. J. Chem. Theory Comput., 9(1) (2013) 338-354.

[53] M. Kubillus, T. Kubar, M. Gaus, J. Rezac, M. Elstner. Parameterization of the DFTB3 Method for Br, Ca, Cl, F, I, K, and Na in Organic and Biological Systems. J. Chem. Theory Comput., 11(1) (2015) 332-342.

[54] J. P. Perdew, J. A. Chevary, S. H. Vosko, K. A. Jackson, M. R. Pederson, D. J. Singh, C. Fiolhais. Atoms, molecules, solids, and surfaces: Applications of the generalized gradient approximation for exchange and correlation. Phys. Rev. B, 46 (1992) 6671.

[55] J. P. Perdew, Y. Wang. Electronic Structure of Solids'91. Academie, Berlin, 1991.

[56] J. Tao, J. P. Perdew, V. N. Staroverov, G. E. Scuseria. Climbing the density functional ladder: nonempirical meta-generalized gradient approximation designed for molecules and solids. Phys. Rev. Lett., 91 (2003) 146401.

[57] V. N. Staroverov, G. E. Scuseria, J. Tao, J. P. Perdew. Comparative assessment of a new nonempirical density functional: Molecules and hydrogen-bonded complexes. J. Chem. Phys., 119 (2003) 12129. 
[58] A. D. Becke. Density-functional thermochemistry. III. The role of exact exchange. J. Chem. Phys., 98(7) (1993) 5648-5652.

[59] C. Lee, W. Yang, R. G. Parr. Development of the Colle-Salvetti correlation-energy formula into a functional of the electron density. Phys. Rev. B, 37 (1988) 785.

[60] P. J. Stephens, F. J. Devlin, C. F. Chabalowski, M. J. Frisch. Ab Initio Calculation of Vibrational Absorption and Circular Dichroism Spectra Using Density Functional Force Fields. J. Phys. Chem., 98(45) (1994) 11623-11627.

[61] Y. Zhao, D. G. Truhlar. A new local density functional for main-group thermochemistry, transition metal bonding, thermochemical kinetics, and noncovalent interactions. J. Chem. Phys., 125 (2006) 194101.

[62] Y. Zhao, D. G. Truhlar. The M06 suite of density functionals for main group thermochemistry, thermochemical kinetics, noncovalent interactions, excited states, and transition elements: two new functionals and systematic testing of four M06-class functionals and 12 other functionals. Theor. Chem. Acc., 120 (2008) 215-241.

[63] M. Seth, T. Ziegler. Range-separated exchange functionals with Slater-type functions. J. Chem. Theor. Comput., 8 (2012) 901-907.

[64] E. van Lenthe, E. J. Baerends. Optimized Slater-type basis sets for the elements 1-118. J. Comput. Chem., 24 (2003) 1142-1156.

[65] C. R. Jacob, S. M. Beyhan, R. E. Bulo, A. S. P. Gomes, A. W. Götz, K. Kiewisch, J. Sikkema, L. Visscher. PyADF - A scripting framework for multiscale quantum chemistry. J. Comput. Chem., 32 (2011) 2328-2338.

[66] A. Lembarki, H. Chermette. Obtaining a gradient-corrected kinetic-energy functional from the Perdew-Wang exchange functional. Phys. Rev. A, 50 (1994) 5328-5331. 
[67] T. A. Wesołowski, J. Weber. Kohn-Sham equations with constrained electron density: An iterative evaluation of the ground-state electron density of interacting molecules. Chem. Phys. Lett., 248 (1996) 71-76.

[68] O. Treutler, R. Ahlrichs. Efficient molecular numerical integration schemes. J. Chem. Phys., 102 (1995) 346.

[69] M. Franchini, P. H. T. Philipsen, L. Visscher. The Becke Fuzzy Cells Integration Scheme in the Amsterdam Density Functional Program Suite. J. Comput. Chem., 34 (2013) 1819.

[70] J. P. Unsleber, T. Dresselhaus, K. Klahr, D. Schnieders, M. Böckers, D. Barton, J. Neugebauer. SEREnity: A subsystem quantum chemistry program. J. Comput. Chem., 39 (2018) 788-798.

[71] C. Herrmann, M. Reiher, B. A. Hess. Comparative analysis of local spin definitions. J. Chem. Phys., 122 (2005) 034102.

[72] C. Herrmann, L. Yu, M. Reiher. Spin States in Polynuclear Clusters: The $\left[\mathrm{Fe}_{2} \mathrm{O}_{2}\right]$ Core of the Methane Monooxygenase Active Site. J. Comput. Chem., 27 (2006) $1223-1239$.

[73] A. J. Cohen, D. J. Tozer, N. C. Handy. Evaluation of $\left\langle\hat{S}^{2}\right\rangle$ in density functional theory. J. Chem. Phys., 126 (2007) 214104.

[74] A. S. Menon, L. Radom. Consequences of Spin Contamination in Unrestricted Calculations on Open-Shell Species: Effect of Hartree-Fock and Møller-Plesset Contributions in Hybrid and Double-Hybrid Density Functional Theory Approaches. J. Phys. Chem. A, 112 (2008) 13225-13230.

[75] T. J. Eisenmayer, H. J. M. de Groot, E. van de Wetering, J. Neugebauer, F. Buda. Mechanism and Reaction Coordinate of Directional Charge Separation in Bacterial Reaction Centers. J. Phys. Chem. Lett., 3 (2012) 694-697. 
[76] A. Alia, E. Roy, P. Gast, H. J. van Gorkom, H. J. M. de Groot, G. Jeschke, J. Matysik. Photochemically induced dynamic nuclear polarization in photosystem I of plants observed by ${ }^{13} \mathrm{C}$ magic-angle spinning NMR. J. Am. Chem. Soc., 126 (2004) 12819 12826.

[77] G. J. Janssen, P. Eschenbach, P. Kurle, B. E. Bode, J. Neugebauer, H. J. M. de Groot, J. Matysik, A. Alia. Analysis of the electronic asymmetry of the primary electron donor of photosystem I of Spirodela oligorrhiza by photo-CIDNP solid-state NMR. submitted to J. Magn. Reson., 1 (2020) 261-274.

[78] L. Krabben, E. Schlodder, R. Jordan, D. Carbonera, G. Giacometti, H. Lee, A. N. Webber, W. Lubitz. Influence of the Axial Ligands on the Spectral Properties of P700 of Photosystem I. A Study of Site-Directed Mutants. Biochemistry, 39(42) (2000) 13012-13025.

[79] M. G. Zysmilich, A. McDermott. Photochemically Induced Dynamic Nuclear Polarization in the Solid-State ${ }^{15} \mathrm{~N}$ Spectra of Reaction Centers from Photosynthetic Bacteria Rhodobacter sphaeroides R-26. J. Am. Chem. Soc., 116 (1994) 8362-8363.

[80] M. G. Zysmilich, A. McDermott. Photochemically Induced Nuclear Spin Polarization in Bacterial Photosynthetic Reaction Centers: Assignments of the ${ }^{15} \mathrm{~N}$ SSNMR Spectra. J. Am. Chem. Soc., 118 (1996) 5867-5873.

[81] S. Prakash, A. Alia, P. Gast, H. J. M. de Groot, G. Jeschke, J. Matysik. Magnetic Field Dependence of Photo-CIDNP MAS NMR on Photosynthetic Reaction Centers of Rhodobacter sphaeroides WT. J. Am. Chem. Soc., 127 (2005) 14290-14298.

[82] S. Paul, U. Roy, M. Böckers, J. Neugebauer, A. Alia, J. Matysik. ${ }^{15}$ N photo-CIDNP MAS NMR analysis of a bacterial photosynthetic reaction center of Rhodobacter sphaeroides wildtype. J. Chem. Phys., 151 (2019) 195101. 
[83] P. J. O’Malley, S. J. Collins. The Effect of Axial Mg Ligation on the Geomery and Spin Density Distribution of Chlorophyll and Bacteriochlorophyll Cation Free Radical Models: A Density Functional Study. J. Am. Chem. Soc., 123 (2001) 1104211046.

[84] A. Alia, P. K. Wawrzyniak, G. J. Janssen, F. Buda, J. Matysik, H. J. M. de Groot. Differential Charge Polarization of Axial Histidines in Bacterial Reaction Centers Balances the Asymmetry of the Special Pair. J. Am. Chem. Soc., 131 (2009) 96269627. 